\title{
Whenever a quantum environment emerges as a classical system, it behaves like a measuring apparatus
}

\author{
Caterina Foti ${ }^{1,2}$, Teiko Heinosaari ${ }^{3}$, Sabrina Maniscalco ${ }^{3}$, and Paola Verrucchi ${ }^{4,1,2}$ \\ ${ }^{1}$ Dipartimento di Fisica e Astronomia, Università di Firenze, I-50019, Sesto Fiorentino (FI), Italy \\ ${ }^{2}$ INFN, Sezione di Firenze, I-50019, Sesto Fiorentino (FI), Italy \\ ${ }^{3}$ QTF Centre of Excellence, Turku Centre for Quantum Physics, Department of Physics and Astronomy, University of Turku, \\ FIN-20014, Turku, Finland \\ ${ }^{4}$ ISC-CNR, at Dipartimento di Fisica e Astronomia, Università di Firenze, I-50019, Sesto Fiorentino (FI), Italy \\ August 6, 2019
}

We study the dynamics of a quantum system $\Gamma$ with an environment $\Xi$ made of $N$ elementary quantum components. We aim at answering the following questions: can the evolution of $\Gamma$ be characterized by some general features when $N$ becomes very large, regardless of the specific form of its interaction with each and every component of $\Xi$ ? In other terms: should we expect all quantum systems with a macroscopic environment to undergo a somehow similar evolution? And if yes, of what type? In order to answer these questions we use well established results from large$N$ quantum field theories, particularly referring to the conditions ensuring a large$N$ quantum model to be effectively described by a classical theory. We demonstrate that the fulfillment of these conditions, when properly imported into the framework of the open quantum systems dynamics, guarantees that the evolution of $\Gamma$ is always of the same type of that expected if $\Xi$ were a measuring apparatus, no matter the details of the actual interaction. On the other hand, such details are found to determine the specific basis w.r.t. which $\Gamma$ undergoes the decoherence dictated by the dynamical description of the quantum measurement process. This result wears two hats: on the one hand it clarifies the physical origin of the formal statement that, under certain conditions, any channel from $\rho_{\Gamma}$ to $\rho_{\Xi}$ takes the form of a measure-and-prepare map, as recently shown in Ref. [1]; on the other hand, it for-

Caterina Foti: caterina.foti@unifi.it malizes the qualitative argument that the reason why we do not observe state superpositions is the continual measurement performed by the environment.

\section{Introduction}

There exist two closely-related questions about the quantum mechanical nature of our universe that keep being intriguing after decades of thought processing: how is it that we do not experience state superpositions, and why we cannot even see them when observing quantum systems. As for the latter question, it is somehow assumed that this is due to the continual measurement process acted upon by the environment. However, despite often being considered as an acceptable answer, this argument is not a formal result, and attempts to make it such have been only recently proposed [1-3]. In fact, the current analysis of the quantum measurement process [4], its Hamiltonian description $[5,6]$, as well as its characterization in the framework of the open quantum systems (OQS) dynamics [7] has revealed the qualitative nature of the above argument, thus making it ever more urgent to develop a rigorous approach to the original question. This is the main goal of our work.

Getting back to the first question, the answer offered by the statement that microscopic systems obey quantum rules while macroscopic objects follow the classical ones, is by now considered unsatisfactory. Macroscopic objects, indeed, may exhibit a distinctive quantum behaviour (as seen for instance in superconductivity, Bose-Einstein condensation, magnetic properties of large molecules with $S=1 / 2$ ), meaning 
that the large- $N$ condition is not sufficient persé for a system made of $N$ quantum particles to behave classically. In fact, there exist assumptions which single out the minimal structure any quantum theory should possess if it is to have a classical limit [8]. Although variously expressed depending on the approach adopted by different authors (see the thorough discussion on the relation between large- $N$ limits and classical theories developed in Sec.VII of Ref. [8]), these assumptions imply precise physical constraints on the quantum theory that describes a macroscopic quantum system if this has to behave classically. In what follows, these assumptions will formally characterize the quantum environment, in order to guarantee that the environment, and it alone, behaves classically. The relevance of the sentence "and it alone" must be stressed: indeed, the work done in the second half of the last century on the $N \rightarrow \infty$ limit of quantum theories is quite comprehensive but it neglects the case when the large- $N$ system is the big partner of a principal quantum system, that only indirectly experiences such limit. This is, however, an exemplary situation in quantum technologies and OQS, hence the questions asked at the beginning of this Introduction have recently been formulated in the corresponding framework [1-3, 9-16].

In this work, we develop an original approach which uses results for the large- $N$ limit of quantum theories in the framework of OQS dynamics. This allows us to show that details of the interaction between a quantum principal system $\Gamma$ and its environment $\Xi$ are irrelevant in determining the main features of the state of $\Xi$ at any time $\tau$ in the large- $N$ limit, as long as such limit implies a classical behaviour for $\Xi$ itself. If this is the case, indeed, such state can always be recognized as that of an apparatus that measures some observable of the principal system. The relation between our findings and the two questions that open this section is evident.

The paper is structured as follows. In the first section we define the dynamical maps characterizing the two evolutions that we aim at comparing. We do so through a parametric representation introduced in Sec. 3. In Sec. 4, we focus on a peculiar property of generalized coherent states, particularly relevant when the large- $N$ limit is considered. As the environment is doomed to be macrocopic and behave classically, we then im- plement such limit in Sec. 5, being finally able to show what we were looking for. In Sec. 6 we comment on the assumptions made, while the results obtained are summed up in the concluding section.

\section{Schmidt decomposition and dynam- ical maps}

We consider the unitary evolution of an isolated bipartite system $\Psi=\Gamma+\Xi$, with Hilbert space $\mathcal{H}_{\Gamma} \otimes \mathcal{H}_{\Xi}$; being $\Psi$ isolated, it is

$$
|\Psi(t)\rangle=e^{-i \hat{H} t}|\Psi\rangle
$$

where $\hbar=1$ and $\hat{H}$ is any Hamiltonian, describing whatever interaction between $\Gamma$ and $\Xi$. The state $|\Psi\rangle$ is assumed separable

$$
|\Psi\rangle=|\Gamma\rangle \otimes|\Xi\rangle
$$

meaning that we begin studying the evolution at a time $t=0$ when both $\Gamma$ and $\Xi$ are in pure states. This is not a neutral assumption, and we will get back to it in Sec. 6 .

At any fixed time $\tau$, there exists a Schmidt decomposition of the state (1),

$$
|\Psi(\tau)\rangle=\sum_{\gamma} c_{\gamma}|\gamma\rangle\left|\xi_{\gamma}\right\rangle
$$

with $\gamma=1, \ldots, \operatorname{dim} \mathcal{H}_{\Gamma}, c_{\gamma} \in \mathbb{R}^{+}$for $\gamma \leq \gamma_{\max } \leq$ $\operatorname{dim} \mathcal{H}_{\Gamma}, c_{\gamma}=0$ for $\gamma>\gamma_{\max }, \sum_{\gamma} c_{\gamma}^{2}=1$, and the symbol $\otimes$ understood (as hereafter done whenever convenient). The states $\{|\gamma\rangle\}_{\mathcal{H}_{\Gamma}}$, and $\left\{\left|\xi_{j}\right\rangle\right\}_{\mathcal{H}_{\Xi}}$ with $j=1, \ldots \operatorname{dim} \mathcal{H}_{\Xi}$, form what we will hereafter call the $\tau$-Schmidt bases, to remind that the Schmidt decomposition is state-specific and therefore depends on the time $\tau$ appearing in the LHS of Eq.(3), in whose RHS we have instead understood the $\tau$-dependence of $c_{\gamma},|\gamma\rangle$, and $\left|R_{\gamma}\right\rangle$, for the sake of a lighter notation. Consistently with the idea that $\Xi$ is a macroscopic system, we take $\gamma_{\max }<\operatorname{dim} \mathcal{H}_{\Xi}$ : therefore, the states $\left\{\left|\xi_{\gamma}\right\rangle\right\}_{\mathcal{H}_{\Xi}}$ entering Eq.(3) are a subset of the pertaining $\tau$-Schmidt basis. Given that $|\Gamma\rangle$ is fully generic, the unitary evolution (1) defines, via $\rho_{\Xi}=\operatorname{Tr}_{\Gamma} \rho_{\Psi}$, the CPTP linear map (from $\Gamma$ to $\Xi$-states)

$$
\mathcal{E}:|\Gamma\rangle\left\langle\Gamma\left|\rightarrow \rho_{\Xi}=\sum_{\gamma} c_{\gamma}^{2}\right| \xi_{\gamma}\right\rangle\left\langle\xi_{\gamma}\right| .
$$


Being the output $\rho_{\Xi}$ a convex sum of orthogonal projectors, Eq.(4) might describe a projective measurement acted upon by $\Xi$ on the principal system $\Gamma$, by what is often referred to as measure-and-prepare (m\&p) map. However, for this being the case, the probability reproducibility condition [17] must also hold, meaning that, given

$$
|\Gamma\rangle=\sum_{\gamma} a_{\gamma}|\gamma\rangle,
$$

it should also be $c_{\gamma}^{2}=\left|a_{\gamma}\right|^{2}, \forall \gamma$. This condition, however, cannot be generally true, if only for the $\tau$-dependence of the Schmidt coefficients $\left\{c_{\gamma}\right\}$ which is not featured by the set $\left\{a_{\gamma}\right\}$. In fact, there exists a dynamical model (the Ozawa's model [5] for projective von Neumann measurement described in Appendix A) for which $c_{\gamma}^{2}=$ $\left|a_{\gamma}\right|^{2}, \forall \gamma$ and $\forall \tau$. Such model is defined by a Hamiltonian where the operators acting on $\Gamma$ must commute with each other, a condition that identifies what we will hereafter dub a measurelike Hamiltonian, $\hat{H}^{\mathrm{M}}$, with the apex M hinting at the corresponding measurement process. The evolution defined by $\exp \left\{-i t \hat{H}^{\mathrm{M}}\right\}$ will be consistently dubbed measure-like dynamics ${ }^{1}$.

Once established that Eq.(4) does not define a $m \& p$ map, we can nonetheless use the elements provided by the Schmidt decomposition as ingredients to construct a measure-like Hamiltonian $\hat{H}^{\mathrm{M}}$ whose corresponding m\&p map, $\mathcal{E}^{\mathrm{M}}$ : $|\Gamma\rangle\langle\Gamma| \rightarrow \rho_{\Xi}^{\mathrm{M}}$ is the "nearest" possible to the actual $\mathcal{E}$, Eq.(4).

To this aim, we first use the $\tau$-Schmidt bases $\{|\gamma\rangle\}_{\mathcal{H}_{\Gamma}}$ and $\left\{\left|\xi_{j}\right\rangle\right\}_{\mathcal{H}_{\Xi}}$ to define the hermitian operators

$$
\hat{O}_{\Gamma}=\sum_{\gamma} \varepsilon_{\gamma}|\gamma\rangle\left\langle\gamma\left|\quad, \quad \hat{O}_{\Xi}=\sum_{j} E_{j}\right| \xi_{j}\right\rangle\left\langle\xi_{j}\right|,
$$

with $\varepsilon_{\gamma}, E_{j}$ arbitrary real numbers; we then write the interaction Hamiltonian

$$
\hat{H}^{\mathrm{M}}=g \hat{O}_{\Gamma} \otimes \hat{O}_{\Xi},
$$

with $g$ some coupling constant, which has the form prescribed by the Ozawa's model (see Appendix A for more details).

\footnotetext{
${ }^{1}$ Giving a Hamiltonian description of more general quantum measurement processes, i.e., identifying the appropriate propagator for the dynamics of such processes up to the output production, is a very relevant problem that has recently attracted the interest of several authors, including some of us.
}

Further using the Schmidt coefficients, we construct the separable state

$$
\left|\Psi^{\mathrm{M}}\right\rangle=|\Gamma\rangle \otimes\left|\Xi^{\mathrm{M}}\right\rangle
$$

where $|\Gamma\rangle$ is the same as in Eq.(2), while $\left|\Xi^{\mathrm{M}}\right\rangle=$ $\sum_{\gamma} c_{\gamma}\left|\xi_{\gamma}\right\rangle$, with $c_{\gamma}$ and $\left|\xi_{\gamma}\right\rangle$ as in Eq.(3). Finally we define

$$
\left|\Psi_{\tau}^{\mathrm{M}}\right\rangle=e^{-i \hat{H}^{\mathrm{M}} \tau}\left|\Psi^{\mathrm{M}}\right\rangle
$$

that reads, using $\hat{O}_{\Gamma}|\gamma\rangle=\varepsilon_{\gamma}|\gamma\rangle, \hat{O}_{\Xi}\left|\xi_{\gamma}\right\rangle=$ $E_{\gamma}\left|\xi_{\gamma}\right\rangle$, and $|\Gamma\rangle=\sum_{\gamma} a_{\gamma}|\gamma\rangle$,

$$
\begin{aligned}
\left|\Psi_{\tau}^{\mathrm{M}}\right\rangle & =e^{-i \hat{H}^{\mathrm{M}} \tau} \sum_{\gamma} a_{\gamma}|\gamma\rangle \sum_{\gamma^{\prime}} c_{\gamma^{\prime}}\left|\xi_{\gamma^{\prime}}\right\rangle \\
& =\sum_{\gamma, \gamma^{\prime}} a_{\gamma}|\gamma\rangle c_{\gamma^{\prime}} e^{-i \varphi_{\gamma \gamma^{\prime}}}\left|\xi_{\gamma^{\prime}}\right\rangle,
\end{aligned}
$$

with $\varphi_{\gamma \gamma^{\prime}} \equiv \tau g \varepsilon_{\gamma} E_{\gamma^{\prime}} \in \mathbb{R}$. Do notice the different notation for the time-dependence in Eqs. (3) and (9): this is to underline that while the former indicates how the state $|\Psi\rangle$ of a system with Hamiltonian $\hat{H}$ evolves into $|\Psi(t)\rangle$ at any time $t$, the latter represents a state whose dependence on $\tau$ not only enters as a proper time in the propagator, but also, as a parameter, in the definition of $\hat{H}^{\mathrm{M}}$ and $\left|\Xi^{\mathrm{M}}\right\rangle$, via the $\tau$-dependence of the Schmidt decomposition (3). Nonetheless, the state $\left|\Psi_{\tau}^{\mathrm{M}}\right\rangle$ can still be recognized as that in which $\Psi$ would be at time $\tau$, were its initial state $\left|\Psi^{\mathrm{M}}\right\rangle$ and its evolution ruled by the measure-like interaction Eq. (7).

Given that $|\Gamma\rangle$ is fully generic, Equation (9) defines, via $\rho_{\Xi}=\operatorname{Tr}_{\Gamma} \rho_{\Psi}$, the CPTP map from $\Gamma$ to $\Xi$-states

$$
\begin{aligned}
\mathcal{E}^{\mathrm{M}} & :|\Gamma\rangle\langle\Gamma| \rightarrow \rho_{\Xi}^{\mathrm{M}}= \\
& =\sum_{\gamma \gamma^{\prime} \gamma^{\prime \prime}}\left|a_{\gamma}\right|^{2} c_{\gamma^{\prime}} c_{\gamma^{\prime \prime}} e^{i\left(\varphi_{\gamma \gamma^{\prime \prime}}-\varphi_{\gamma \gamma^{\prime}}\right)}\left|\xi_{\gamma^{\prime}}\right\rangle\left\langle\xi_{\gamma^{\prime \prime}}\right| .
\end{aligned}
$$

Notice that $\mathcal{E}^{\mathrm{M}}$ depends on $\tau$ directly, via $\varphi_{\gamma \gamma^{\prime}} \propto$ $\tau$, and indirectly, via the $\tau$-dependence of the Schmidt decomposition, that is of the coefficients $c_{\gamma}$ and the states $\left|\xi_{\gamma}\right\rangle$. Comparing Eqs.(4) and (11) we see that $\mathcal{E}^{\mathrm{M}}$ has the right coefficients $\left\{\left|a_{\gamma}\right|^{2}\right\}$ but the wrong form, i.e., it is not a sum of orthogonal projectors, while $\mathcal{E}$ has the correct form but with the wrong coefficients, $\left\{c_{\gamma}^{2}\right\}$. In fact, were these two maps equal in some limit, it would mean the following: for each time $\tau$, there exists an observable for $\Gamma$, (depending on $\tau$ itself) 
such that the state into which $\Xi$ has evolved due to its true interaction with $\Gamma$ is the same, in such limit, as if $\Xi$ itself were some measuring apparatus proper to that observable, which is quite a statement. Since $\mathcal{E}$ and $\mathcal{E}^{\mathrm{M}}$ are linear, they are the same map iff the output states $\rho_{\Xi}$ and $\rho_{\Xi}^{\mathrm{M}}$ are equal for whatever input $|\Gamma\rangle$. We can therefore concentrate upon the structure of such output states, which we will do in the next section by introducing a proper parametric representation.

\section{Parametric representation with envi- ronmental coherent states}

The parametric representation with environmental coherent states (PRECS) is a theoretical tool that has been recently introduced [18, 19] to specifically address those bipartite quantum systems where one part, on its own made by $N$ elementary components, shows an emerging classical behaviour in the large- $N$ limit $[6,20-23]$. The method makes use of generalized coherent states (GCS) for the system intended to become macroscopic.

The construction of GCS, sometimes referred to as group-theoretic, goes as follows [24]. Associated to any quantum system there is a Hilbert space $\mathcal{H}$ and a dynamical group $\mathcal{G}$, which is the group containing all the propagators that describe possible evolutions of the system (quite equivalently, $\mathcal{G}$ is the group corresponding to the Lie algebra $\mathfrak{g}$ to which all the physical Hamiltonians of the system belong). Once these ingredients are known, a reference state $|0\rangle$ is arbitrarily chosen in $\mathcal{H}$ and the subgroup $\mathcal{F}$ of the propagators that leave such state unchanged (apart from an irrelevant overall phase) is determined. This is usually referred to as the stability subgroup. Elements $\hat{\omega}$ of $\mathcal{G}$ that do not belong to such subgroup, $\hat{\omega} \in \mathcal{G} / \mathcal{F}$, generate the GCS upon acting on the reference state, $\hat{\omega}|0\rangle=|\omega\rangle$, and are usually dubbed "displacement" operators. The GCS construction further entails the definition of an invariant ${ }^{2}$ measure $d \mu(\hat{\omega})$ on $\mathcal{G} / \mathcal{F}$ such that a resolution of the identity on $\mathcal{H}$ is provided in the form

$$
\int_{\mathcal{G} / \mathcal{F}} d \mu(\hat{\omega})|\omega\rangle\langle\omega|=\hat{\mathbb{I}}_{\mathcal{H}}
$$

\footnotetext{
${ }^{2}$ The measure $d \mu(\hat{\omega})$ is called invariant because it is
} left unchanged by the action of $\mathcal{G}$.

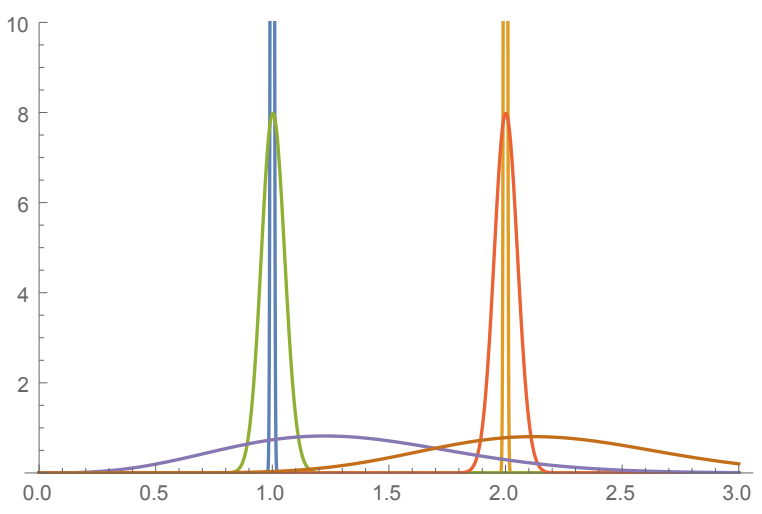

Figure 1: $\quad|\langle\alpha \mid n\rangle|^{2}$ as a function of $\sqrt{\alpha \alpha^{*}}$, with $n=1$ (left) and $n=4$ (right), for $N=1,10,1000$ (bottom to top).

One of the most relevant byproduct of the GCS construction is the definition of a differentiable manifold $\mathcal{M}$ via the chain of one-to-one correspondences

$$
\hat{\omega} \subset \mathcal{G} / \mathcal{F} \Leftrightarrow|\omega\rangle \in \mathcal{H} \Leftrightarrow \omega \subset \mathcal{M},
$$

so that to any GCS is univoquely associated a point on $\mathcal{M}$, and viceversa. A measure $d \mu(\omega)$ on $\mathcal{M}$ is consistently associated to the above introduced $d \mu(\hat{\omega})$, so that requiring GCS to be normalized, $\langle\omega \mid \omega\rangle=1$, implies

$$
\begin{aligned}
\langle\omega \mid \omega\rangle & =\left\langle\omega\left|\left[\int_{\mathcal{G} / \mathcal{F}} d \mu(\hat{\omega})|\omega\rangle\langle\omega|\right]\right| \omega\right\rangle \\
& =\int_{\mathcal{M}} d \mu(\omega)|\langle\omega \mid \omega\rangle|^{2}=1 ;
\end{aligned}
$$

notice that GCS are not necessarily orthogonal.

One important aspect of the GCS construction is that it ensures the function $\langle\omega|\rho| \omega\rangle$ for whatever state $\rho$ (often called Husimi function in the literature $^{3}$ ) is a well-behaved probability distribution on $\mathcal{M}$ that uniquely identifies $\rho$ itself. As a consequence, studying $\langle\omega|\rho| \omega\rangle$ on $\mathcal{M}$ is fully equivalent to perform a state-tomography of $\rho$ on the Hilbert space, and once GCS are available one can analyze any state $\rho$ of the system by studying its Husimi function on $\mathcal{M}$, which is what we will do in the following. We refer the reader to Refs. [24, 25] for more details.

\footnotetext{
${ }^{3}$ In fact, a "Husimi function" is in principle defined on a classical phase-space, while $\mathcal{M}$ is a differential manifold with a simplectic structure that should not be considered a phase-space, yet, i.e., before the large- $N$ limit is taken; however, it is quite conventional to extend the term to the expectation value of $\rho$ on GCS.
} 
When GCS are relative to a system $\Xi$ which is the environment of a principal system $\Gamma$, we call them Environmental Coherent States (ECS).

Getting back to the setting of section 2, we first recognize that, if they were to represent different evolutions of the same physical system, the propagators $\exp \{-i \hat{H} \tau\}$ and $\exp \left\{-i \hat{H}^{\mathrm{M}} \tau\right\}$ must belong to the same dynamical group, as far as their action on $\mathcal{H}_{\Xi}$ is concerned. More explicitely, this group is identified as follows: $i$ ) consider all the operators acting on $\mathcal{H}_{\Xi}$ in the total Hamiltonians $\hat{H}$ and $\left.\hat{H}^{M} ; i i\right)$ find the algebra to which they all belong (notice that, as both Hamiltonians refer to the same physical system, the above operators must belong to the same algebra $\mathfrak{g} ; \mathrm{iii}$ ) recognize the dynamical group as that associated to the above algebra $\mathfrak{g}$ via the usual exponential Lie map (for several examples see for instance Refs. [6, 18, 22, 23]). This is the group to be used for constructing the ECS, according to the procedure briefly sketched above. Once ECS are constructed, the PRECS of any pure state $|\psi\rangle$ of $\Psi$ is obtained by inserting an identity resolution in the form (12) into any decomposition of $|\psi\rangle$ as linear combination of separable (w.r.t. the partition $\Psi=\Gamma+\Xi)$ states. Explicitly, one has

$$
|\psi\rangle=\int_{\mathcal{M}} d \mu(\omega) \chi(\omega)|\omega\rangle|\Gamma(\omega)\rangle
$$

where $|\Gamma(\omega)\rangle$ is a normalized state for $\Gamma$ that parametrically depends on $\omega$, while $\chi(\omega)$ is a real function on $\mathcal{M}$ whose square

$$
\chi(\omega)^{2}=\left\langle\omega\left|\rho_{\Xi}\right| \omega\right\rangle,
$$

is the environmental Husimi function relative to $\rho_{\Xi}=\operatorname{Tr}_{\Gamma}|\psi\rangle\langle\psi|$, i.e., the normalized distribution on $\mathcal{M}$ that here represents the probability for the environment $\Xi$ to be in the GCS $|\omega\rangle$ when $\Psi$ is in the pure state $|\psi\rangle$. The explicit form of $\chi(\omega)$ and $|\Gamma(\omega)\rangle$ is obtained from any decomposition of $|\psi\rangle$ into a linear combination of separable (w.r.t. the partition $\Gamma+\Xi)$ states.

In particular, for the states (3) and (10), it is

$$
\chi(\omega)^{2}=\sum_{\gamma} c_{\gamma}^{2}\left|\left\langle\omega \mid \xi_{\gamma}\right\rangle\right|^{2},
$$

and

$$
\begin{aligned}
& \chi^{\mathrm{M}}(\omega)^{2}= \\
& =\sum_{\gamma \gamma^{\prime} \gamma^{\prime \prime}}\left|a_{\gamma}\right|^{2} c_{\gamma^{\prime}} c_{\gamma^{\prime \prime}} e^{i\left(\varphi_{\gamma \gamma^{\prime \prime}}-\varphi_{\gamma \gamma^{\prime}}\right)}\left\langle\omega \mid \xi_{\gamma^{\prime}}\right\rangle\left\langle\xi_{\gamma^{\prime \prime}} \mid \omega\right\rangle,
\end{aligned}
$$

respectively.

Comparing $\chi(\omega)^{2}$ and $\chi^{\mathrm{M}}(\omega)^{2}$ is equivalent to compare $\rho_{\Xi}$ and $\rho_{\Xi}^{\mathrm{M}}$, and hence the maps (4) and (11). However, despite the very specific construction leading to $\left|\Psi_{\tau}^{\mathrm{M}}\right\rangle$, we cannot yet make any meaningful specific comparison between $\chi(\omega)^{2}$ and $\chi^{\mathrm{M}}(\omega)^{2}$ at this stage. Indeed, we still have to exploit the fact that the environment is doomed to be big and behave classically, which is why ECS turn out to be so relevant to the final result, as shown in the next section.

\section{Large- $N$ and classical limit}

As mentioned in the Introduction, a physical system which is made by a large number $N$ of quantum constituents does not necessarily obey the rules of classical physics. However, several authors $[8,24,26,27]$ have shown that if GCS exist and feature some specific properties, then the structure of a classical theory $\mathcal{C}$ emerges from that of a quantum theory $\mathcal{Q}$. In particular, the existence of GCS establishes a relation between the Hilbert space of $\mathcal{Q}$ and the manifold $\mathcal{M}$ that their construction implies, which turns out to be the phase-space of the classical theory that emerges as the large- $N$ limit of $\mathcal{Q}$. In fact, one should rather speak about the $k \rightarrow 0$ limit of $\mathcal{Q}$, with $k$ the real positive number, referred to as "quanticity parameter", such that all the commutators of the theory (or anticommutators, in the fermionic case) vanish with $k$. However, all known quantum theories for systems made by $N$ components have $k \sim \frac{1}{N^{p}}$ with $p$ a positive number: therefore, for the sake of clarity, we will not hereafter use the vanishing of the quanticity parameters but rather refer to the large- $N$ limit (see Appendix B for more details).

Amongst the above properties of GCS, that are thoroughly explained and discussed in Ref. [8] as the assumptions guaranteeing the large- $N$ limit to define a classical theory, one that plays a key role in this work regards the overlaps $\langle\omega \mid \xi\rangle$, whose square modulus represents the probability that a system in some generic pure state $|\xi\rangle$ be observed in the coherent state $|\omega\rangle$. These overlaps never vanish for finite $N$, due to the overcompleteness of GCS: as a consequence, if one considers two orthonormal states, say $\left|\xi^{\prime}\right\rangle$ and $\left|\xi^{\prime \prime}\right\rangle$, there might be a finite probability for a system in a GCS $|\omega\rangle$ to be observed either in $\left|\xi^{\prime}\right\rangle$ or in $\left|\xi^{\prime \prime}\right\rangle$. This formally 

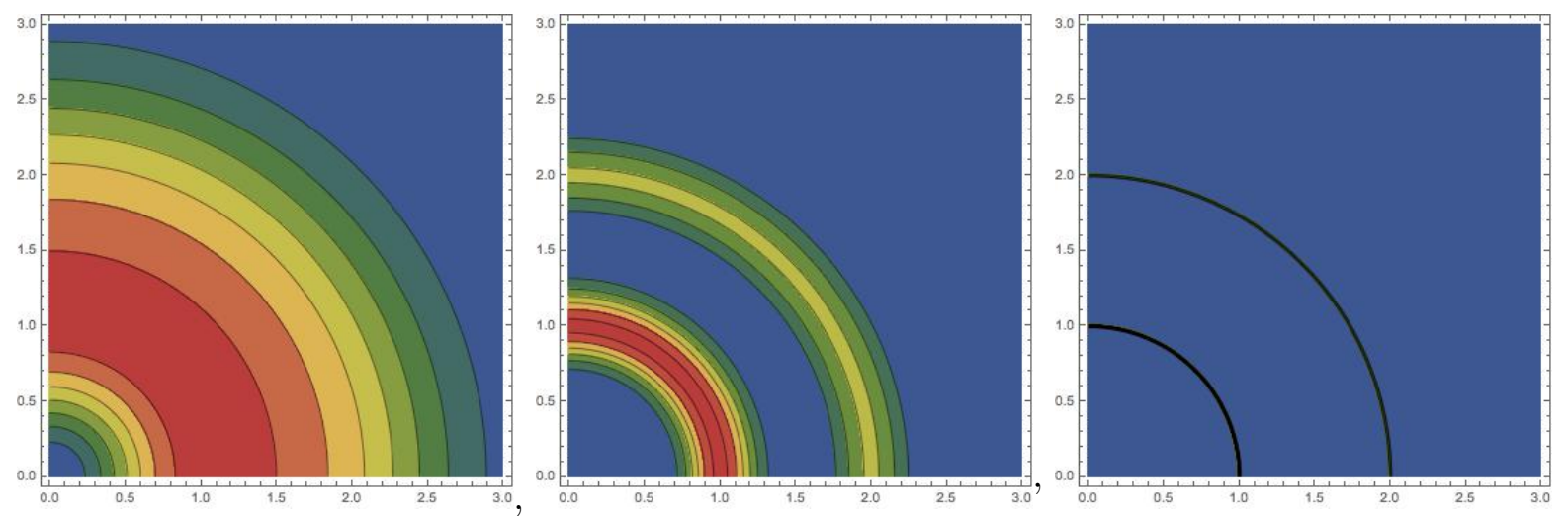

Figure 2: Sum $\left|\left\langle\alpha \mid n^{\prime}\right\rangle\right|^{2}+\left|\left\langle\alpha \mid n^{\prime \prime}\right\rangle\right|^{2}$ with $n^{\prime}=1$ and $n^{\prime \prime}=4$ for $N=1,10,1000$ (left to right): Contourplot on part of $\mathcal{M}$, which is now the complex plane (values increase from blue to red).

implies that, defined $S_{\xi}$ the set of points on $\mathcal{M}$ where $|\langle\omega \mid \xi\rangle|>0$, it generally is $S_{\xi^{\prime}} \cap S_{\xi^{\prime \prime}} \neq \emptyset$.

On the other hand, the quantity

$$
\lim _{N \rightarrow \infty}|\langle\omega \mid \xi\rangle|^{2}
$$

features some very relevant properties. First of all, if $|\xi\rangle$ is another GCS, say $\left|\omega^{\prime}\right\rangle$, the square modulus $\left|\left\langle\omega \mid \omega^{\prime}\right\rangle\right|^{2}$ exponentially vanishes with $\left|\omega-\omega^{\prime}\right|^{2}$ in such a way that the limit (19) converges to the Dirac distribution $\delta\left(\omega-\omega^{\prime}\right)$, thus restoring a notion of distinguishability between different GCS in the large- $N$ limit. Moreover, in Appendix $\mathrm{C}$ we demonstrate that

$$
\left\langle\xi^{\prime} \mid \xi^{\prime \prime}\right\rangle=\delta_{\xi^{\prime} \xi^{\prime \prime}} \Leftrightarrow \lim _{N \rightarrow \infty} S_{\xi^{\prime}} \cap S_{\xi^{\prime \prime}}=\emptyset
$$

meaning that orthonormal states are put together by distinguishable sets of GCS. In other terms, the large- $N$ limit enforces the emergence of a oneto-one correspondence between elements of any orthonormal basis $\{|\xi\rangle\}$ and disjoint sets of GCS, in such a way that the distinguishability of the former is reflected into the disjunction of the latter. Given the relevance of Eq.(20) to this work, let us discuss its meaning with two explicit examples.

\subsection{Field Coherent States}

Consider a system $\Xi$ whose Lie algebra is $\mathfrak{h}_{4}$, i.e., the vector space spanned by $\left\{\hat{a}, \hat{a}^{\dagger}, \hat{n} \equiv \hat{a}^{\dagger} \hat{a}, \hat{\mathbb{I}}\right\}$, with Lie brackets $\left[\hat{a}, \hat{a}^{\dagger}\right]=1$, and $\left[\hat{a}^{(\dagger)}, \hat{n}\right]=$ $(-) \hat{a}^{(\dagger)}$. In order to identify the quanticity parameter $k$, i.e., the parameter whose vanishing makes the Lie brackets of the theory go to zero, one can restore dimensionful ladder operators, $\hat{a}^{(\dagger)} \rightarrow \sqrt{\frac{2 \hbar}{M \omega}} \hat{a}^{(\dagger)}$, and observe that all the commutators vanish in the large- $M$ limit. Further taking $M \propto N$, meaning that the total mass of $\Xi$ is the sum of the masses of the elementary components, which are assumed to have the same mass for the sake of simplicity, it is easily found that $k \sim 1 / N$. As for the GCS , they are the well known field coherent states $\{|\alpha\rangle\}$, with $|0\rangle: \hat{a}|0\rangle=0$ the reference state, and $\mathcal{M}$ the complex plane. The eigenstates of $\hat{n}$ are the Fock states $\{|n\rangle\}$, and $\exp \left\{\alpha \hat{a}-\alpha^{*} \hat{a}^{\dagger}\right\} \equiv \hat{\alpha}$ is the displacement operator such that $|\alpha\rangle=\hat{\alpha}|0\rangle$.

As for the overlaps entering Eq.(18), let us first consider the case when the states $\left\{\left|\xi_{\gamma}\right\rangle\right\}$ are Fock states. In Fig. 1 we show $|\langle\alpha \mid n\rangle|^{2}$ as a function of $|\alpha|^{2}$, for $n=1,2$ and different values of $N$. It is clearly seen that $S_{n^{\prime}} \cap S_{n^{\prime \prime}} \rightarrow \emptyset$ as $N \rightarrow \infty$, meaning that the product of overlaps in Eq.(18) vanishes unless $\gamma^{\prime}=\gamma^{\prime \prime}$, i.e. $n^{\prime}=n^{\prime \prime}$ in this specific example. In order to better visualize $S_{n^{\prime}}$ and $S_{n^{\prime \prime}}$ on $\mathcal{M}$, in Fig. 2 we contour-plot the sum $|\langle\alpha \mid 1\rangle|^{2}+|\langle\alpha \mid 2\rangle|^{2}$ : indeed we see that, as $N$ increases, $S_{1}$ and $S_{2}$ do not intersect. Notice that increasing $N$ does not squeeze $S_{n}$ to the neighbourghood of some point on $\mathcal{M}$, as is the case for $\lim _{N \rightarrow \infty}\left|\left\langle\alpha \mid \alpha^{\prime}\right\rangle\right|^{2}=\delta\left(\alpha-\alpha^{\prime}\right)$, but rather to that of the circle $|\alpha|^{2}=n$. In other terms, more field coherent states overlap with the same Fock state, but different Fock states overlap with distinct sets of field coherent states, in the large- $N$ limit. This picture holds not only for Fock states but, as expressed by Eq.(20), for any pair of orthonormal states. In Fig. 3, for instance, we contour-plot the $\operatorname{sum}|\langle\alpha \mid+\rangle|^{2}+\left.\langle\alpha \mid-\rangle\right|^{2}$ with $| \pm\rangle \equiv(|1\rangle \pm|2\rangle) / \sqrt{2}$ : in this case $S_{+}$and $S_{-}$are disjoint already for $N=1$, and keep shrinking as $N$ increases. 

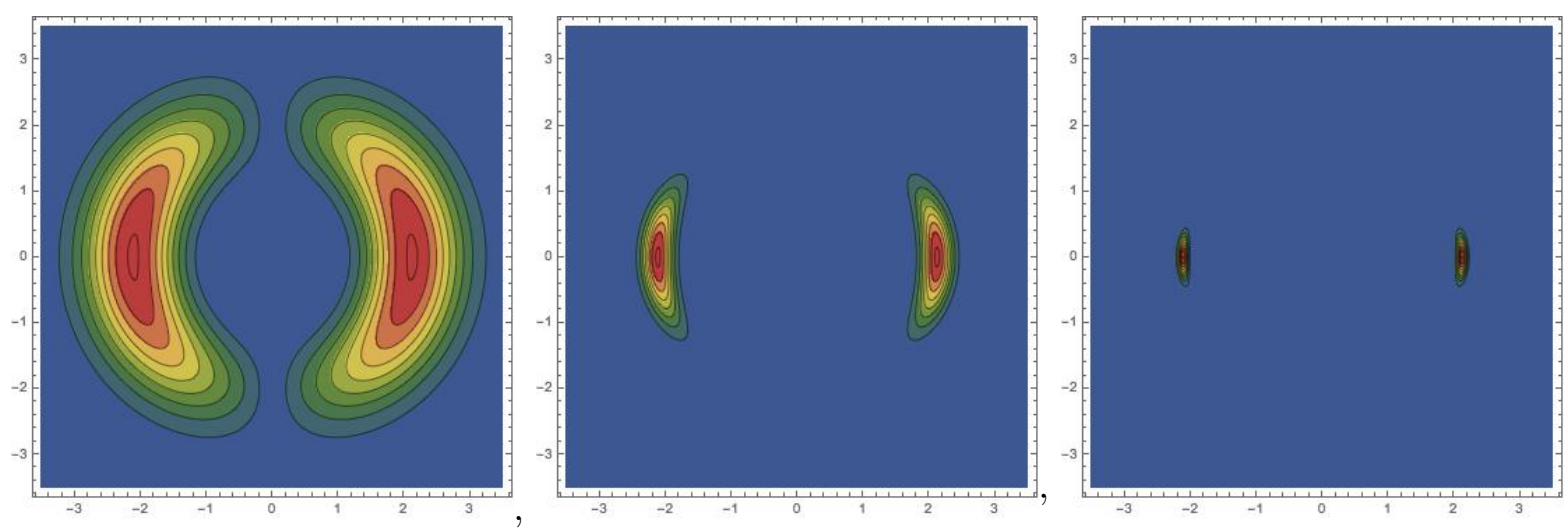

Figure 3: Sum $|\langle\alpha \mid+\rangle|^{2}+|\langle\alpha \mid-\rangle|^{2}$ with $| \pm\rangle=(|1\rangle \pm|2\rangle) / \sqrt{2}$, for $N=1,10,1000$ (left to right): Contourplot on $\mathcal{M}$, which is now the complex plane (values increase from blue to red).

\subsection{Spin Coherent States}

A very similar scenario appears when studying a system $\Xi$ whose Lie algebra is $\mathfrak{s u}(2)$, i.e., the vector space spanned by $\left\{\hat{S}^{+}, \hat{S}^{-}, \hat{S}^{z}\right\}$, with Lie brackets $\left[\hat{S}^{+}, \hat{S}^{-}\right]=2 \hat{S}^{z},\left[\hat{S}^{z}, \hat{S}^{ \pm}\right]= \pm \hat{S}^{ \pm}$, and $|\hat{\mathbf{S}}|^{2}=S(S+1)$, with $S$ fixed and constant; in this case the quanticity parameter is identified by noticing that the normalized operators $\hat{s}^{*} \equiv \frac{1}{S} \hat{S}^{*}, *=z, \pm$, have vanishing commutators in the large- $S$ limit. Further taking $S \propto N$, meaning that the total spin of $\Xi$ is a conserved quantity, whose value is the sum of the spins of each individual component, it is easily found that $k \sim 1 / N$. As for the GCS, they are the so-called spin (or atomic) coherent states $\{|\Omega\rangle\}$, with the reference state $|0\rangle: \hat{S}^{z}|0\rangle=-S|0\rangle$, and $\mathcal{M}$ the unit sphere. The eigenstates of $\hat{S}^{z}$ are $\{|m\rangle\}: \hat{S}^{z}|m\rangle=(-S+m)|m\rangle$, and the displacement operators are $\hat{\Omega}=\exp \left\{\eta \hat{S}^{-}-\eta^{*} \hat{S}^{+}\right\}$, with $\eta=\frac{\theta}{2} e^{i \phi}$, and $\theta \in[0, \pi], \phi \in[0,2 \pi)$ the spherical coordinates. As for the overlaps entering Eq.(18), the analytical expression for $\langle\Omega \mid m\rangle$ is available (see for instance Ref. [24]), which allows us to show, in Fig. 4, the square modulus $|\langle\Omega \mid m\rangle|^{2}$ for $m^{\prime} / S=0.8$ and $m^{\prime \prime} / S=0.4$, for different values of $N$. Again we see that $S_{m^{\prime}} \cap S_{m^{\prime \prime}} \rightarrow \emptyset$ as $N \rightarrow \infty$, implying that the product in Eq.(18) vanishes unless $\gamma^{\prime}=\gamma^{\prime \prime}$, i.e., $m^{\prime}=m^{\prime \prime}$ in this specific example. In Fig. 5 we show the sum $\left|\left\langle\Omega \mid m^{\prime}\right\rangle\right|^{2}+\left|\left\langle\Omega \mid m^{\prime \prime}\right\rangle\right|^{2}$ as density-plot on part of the unit sphere: besides the expected shrinking of the regions where the overlaps are finite, we notice that, as seen in the bosonic case, the support of $\lim _{N \rightarrow \infty}|\langle\Omega \mid m\rangle|^{2}$ does not shrink into the neighbourghood of a point on the sphere, as is the

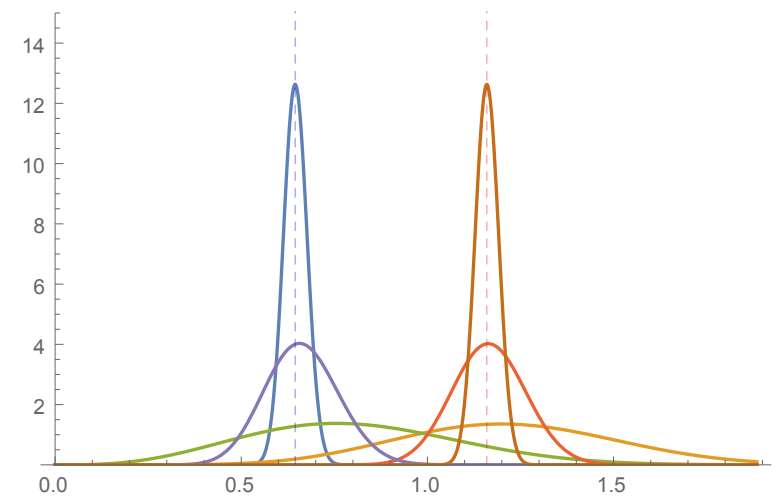

Figure 4: $\quad|\langle\Omega \mid m\rangle|^{2}$ as a function of $\theta$, for $m / S=0.8$ (left) and 0.4 (right), for $N=10,100,1000$ (bottom to top).

case for $\lim _{N \rightarrow \infty}\left|\left\langle\Omega \mid \Omega^{\prime}\right\rangle\right|^{2}=\delta\left(\Omega-\Omega^{\prime}\right)$, but rather into that of the parallel $\cos \theta=m / S$.

\section{A macroscopic environment that be- haves classically}

Let us now get back to the general case and to Eq.(18): the states $\left|\xi_{\gamma^{\prime}}\right\rangle$ and $\left|\xi_{\gamma^{\prime \prime}}\right\rangle$ are othonormal by definition, being elements of the $\tau$-Schmidt basis $\left\{\left|\xi_{j}\right\rangle\right\}_{\mathcal{H}_{\Xi}}$ introduced in Sec.2. Therefore Eq.(20) holds, meaning

$$
\lim _{N \rightarrow \infty}\left\langle\omega \mid \xi_{\gamma^{\prime}}\right\rangle\left\langle\xi_{\gamma^{\prime \prime}} \mid \omega\right\rangle=\lim _{N \rightarrow \infty}\left|\left\langle\omega \mid \xi_{\gamma^{\prime}}\right\rangle\right|^{2} \delta_{\gamma^{\prime} \gamma^{\prime \prime}}
$$

and hence

$$
\lim _{N \rightarrow \infty} \chi^{\mathrm{M}}(\omega)^{2}=\sum_{\gamma \gamma^{\prime}}\left|a_{\gamma}\right|^{2} c_{\gamma^{\prime}}^{2} \lim _{N \rightarrow \infty}\left|\left\langle\omega \mid \xi_{\gamma^{\prime}}\right\rangle\right|^{2} .
$$



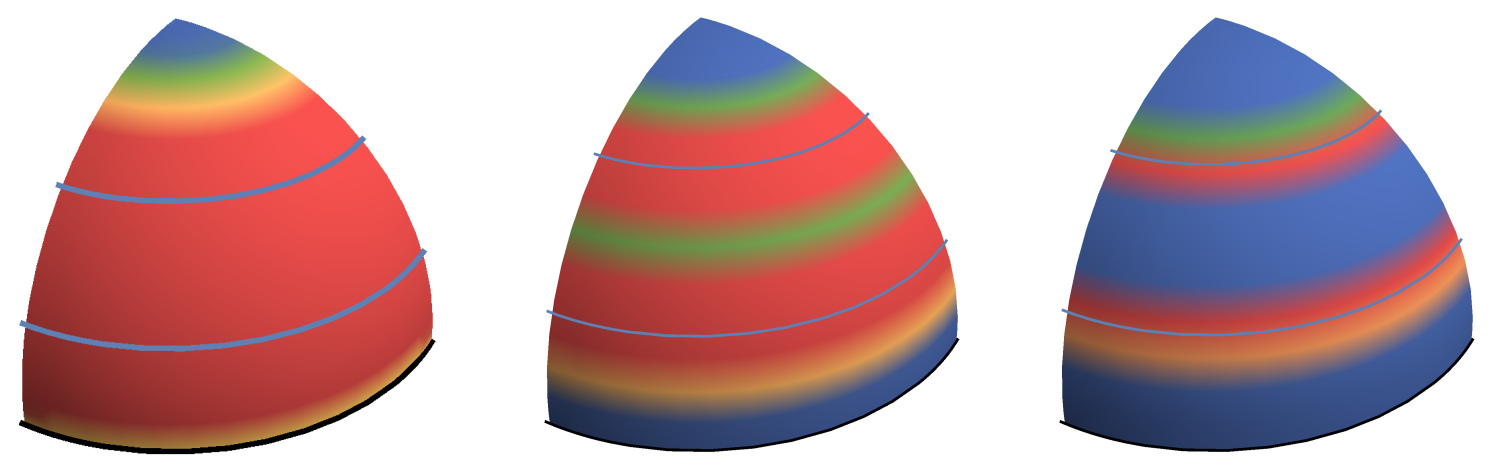

Figure 5: Sum $\left|\left\langle\Omega \mid m^{\prime}\right\rangle\right|^{2}+\left|\left\langle\Omega \mid m^{\prime \prime}\right\rangle\right|^{2}$ with $m^{\prime} / S=0.8$ and $m^{\prime \prime} / S=0.4$, for $N=10,100,1000$ (left to right): Densityplot on part of $\mathcal{M}$, which is now the unit sphere (values increase from blue to red).

Using $\sum_{\gamma}\left|a_{\gamma}\right|^{2}=1$, and the swap $\gamma^{\prime} \leftrightarrow \gamma$, we finally obtain

$$
\lim _{N \rightarrow \infty} \chi^{\mathrm{M}}(\omega)^{2}=\lim _{N \rightarrow \infty} \chi(\omega)^{2}
$$

which is what we wanted to prove, namely that the the dynamical maps (4) and (11) are equal when $\Xi$ is a quantum macroscopic system whose behaviour can be effectively described classically.

\section{Discussion}

Aim of this section is to comment upon some specific aspects of our results, with possible reference to the way other authors have recently tackled the same subject. Let us first consider the assumption that the initial state (2) of the total system $\Psi=\Gamma+\Xi$ be separable. If this is not the case, as it may happen, one must look for the different partition $\Psi=A+B$, such that $|\Psi\rangle=|A\rangle \otimes|B\rangle$. If this partition is still such that the subsystem $B$ is macroscopic and behaves classically, the change is harmless and the whole construction can be repeated with $A$ the quantum system being observed and $B$ its observing environment. On the other hand, if the new partition is such that neither $A$ nor $B$ meet the conditions for being a classical environment, then the problem reduces to the usual one of studying the dynamics of two interacting quantum systems, for which any approach based on effective descriptions is incongrous, as details of the true Hamiltonian will always be relevant. Notice that this analysis is fully consistent with the results presented in Ref. [1], which are embodied into inequalities whose meaning wears off as $\operatorname{dim} \mathcal{H}_{B}$ diminishes. The case when $\Psi$ is not initially in a pure state is similarly tackled by enlarging $\Psi \rightarrow \widetilde{\Psi}$ as much as necessary for $\widetilde{\Psi}$ to be in a pure state: a proper choice of a new partition of $\widetilde{\Psi}$ will follow.

We then want to clarify in what sense the Hamiltonian (7) is said to induce a "measure-like dynamics" or, which is quite equivalent, the channel (11) to define a m\&p map: the quotes indicate that the actual output production, which happens at a certain time according to some process whose nature we do not discuss, is not considered and it only enters the description via the requirement that the probability for each output is the one predicted by Born's rule. To this respect, one might also ask what is the property of $\Gamma$ which is observed by $\Xi$ : this is the one represented, in the Ozawa's model, by the operator $\hat{O}_{\Gamma}$, and it therefore depends on the true evolution via the Schmidt decomposition of the evolved state. To put it another way, details of the interaction do not modify the measure-like nature of the dynamics in the large- $N$ limit, but they do affect what actual measurement is performed by the environment.

Let us now discuss possible connections between our results and Quantum Darwinism [2, 1]. As mentioned at the end of Appendix B, a sufficient condition for a quantum theory to have a large- $N$ limit which is a classical theory is the existence of a global symmetry, i.e., such that its group-elements act non-trivially upon the Hilbert space of each and every component of the total system $\Xi$ that the theory describes. In fact, few simple examples show that quantum theories with different global symmetries can flow 
into the same classical theory in the large- $N$ limit: in other words, echoing L. G. Yaffe in Ref. [8], different quantum theories can be "classically equivalent". If one further argues that amongst classically equivalent quantum theories there always exists a free theory, describing $N$ non-interacting subsystems, it is possible to show that each macroscopic fragment of $\Xi$ can be effectively described as if it were the same measurement apparatus. Work on this point is in progress, based on the quantum de Finetti theorem, results from Refs. [10, 1], and the preliminary analysis reported in Refs. [28, 29]. We close this section by mentioning the possible connection between our description and the way the notion of "objective information" is seen to emerge in Ref. [3]: in fact, the idea that there may be no quantum-to-classical transition involved in the perception of the world around us, that might rather emerge just as a reflection of some specific properties of the underlying quantum states, seems to be consistent with the discussion reported above, and we believe that further investigation on this point might be enlightening.

\section{Conclusions}

The idea that the interaction with macroscopic environments causes the continual statereduction of any quantum system is crucial for making sense of our everyday experience w.r.t. the quantum description of nature. However, the formal analysis of this idea has been unsatisfactory for decades, due to several reasons, amongst which we underline the following.

Firstly the generality of the above idea implies that assumptions on the initial state of the quantum system, and the specific form of the interaction with its environment, should not be made. Secondly, formal tools must be devised to allow the study of the system-plus-environment dynamics in a way that guarantees a genuinely quantum description of the system throughout the crossover of the environment towards a classical behaviour. Finally, a clean procedure is required to ensure that the above crossover takes place when the environment becomes macroscopic, i.e., in the large- $N$ limit of the quantum theory that describes it.

In this work, reminding that principal system and environment are dubbed $\Gamma$ and $\Xi$, respec- tively, we have addressed the above three issues as follows. As for the first point, the analysis is developed by comparing CPTP linear maps from $\Gamma$ - to $\Xi$-states, that do not depend on the initial state of $\Gamma$ by definition. The considered maps, Eqs. (4) and (11), are defined using ingredients provided by the Schmidt decomposition of the system-plus-environment evolved state, Eq. (1), that exists at any time, and whatever the form of the interaction between $\Gamma$ and $\Xi$ is. Regarding the second issue, we have used a parametric representation of the overall system state, Eq. (15), that resorts to generalized coherent states (i.e., coherent states as defined via the group-theoretical approach) for describing $\Xi$. This representation, both for its parametric nature and the peculiar properties of coherent states when the quantumto-classical crossover is considered, allows us to implement the large- $N$ limit for $\Xi$ without making assumptions on $\Gamma$ or affecting its quantum character. The third point has been tackled by using results from large- $N$ quantum field theories: these results provided us with formal conditions that generalized coherent states must fulfill, particularly Eq. (26), in order to describe a macroscopic system that behaves classically.

After this elaboration, we have managed to compare the map defined by the true evolution of $\Gamma+\Xi$, Eq. (4), with that corresponding to a measure-and-prepare dynamical process, Eq. (11), in terms of the difference between probability functions entering the parametric representation, Eqs. (17) and (18). These functions have been demonstrated to become equal when the large- $N$ limit defines a classical dynamics for $\Xi$.

Overall, our approach allows one to tackle the so-called quantum to classical crossover [30] by a rigorous mathematical formulation that provides a physically intuitive picture of the underlying dynamical process. In fact, exploiting the most relevant fact that not every theory has a classical limit, we have shown that any dynamics of whatever OQS defines a Hamiltonian model that characterizes its environment as a measuring apparatus if the conditions ensuring that the above classical limit exists and corresponds to a large$N$ condition upon the environment itself are fulfilled. In other words, if some dynamics emerges in the classical world, it necessarily is a measurelike one. 
Let us conclude by briefly commenting upon the already mentioned phenomenon known as Quantum Darwinism, introduced in [2] and recently considered in [1] from an information theoretic viewpoint. Our work suggests that Quantum Darwinism might emerge as a dynamical process, with its generality due to the versatilility of the Hamiltonian model for the quantum measurement process, and the loss of resolution inherent in the classical description.

\section{Acknowledgments}

CF acknowledges M. Piani and M. Ziman for useful and stimulating discussions. SM and TH acknowledge financial support from the Academy of Finland via the Centre of Excellence program (Project no. 312058) as well as Project no. 287750. CF and PV acknowledge financial support from the University of Florence in the framework of the University Strategic Project Program 2015 (project BRS00215). PV acknowledges financial support from the Italian National Research Council (CNR) via the "Short term mobility" program STM-2015, and declares to have worked in the framework of the Convenzione Operativa between the Institute for Complex Systems of CNR and the Department of Physics and Astronomy of the University of Florence. Finally, CF and PV warmly thank the Turku Centre for Quantum Physics for the kind hospitality.

\section{References}

[1] F.G.S.L. Brandao, M. Piani, and P. Horodecki. Generic emergence of classical features in quantum darwinism. Nature Communications, 6:7908, 2015. DOI: 10.1038/ncomms8908.

[2] W. H. Zurek. Quantum darwinism. Nature Physics, 5:181, 2009. DOI: 10.1038/nphys1202.

[3] R. Horodecki, J.K. Korbicz, and P. Horodecki. Quantum origins of objectivity. Phys. Rev. A, 91:032122, 2015. DOI: 10.1103/PhysRevA.91.032122.

[4] P. Busch, P. Lahti, J. P. Pellonp, and K. Ylinen. Quantum Measurement. Springer Publishing Company, Incorporated, 1st edition,
2016. ISBN 3319433873, 9783319433875. DOI: $10.1007 / 978-3-319-43389-9$.

[5] M. Ozawa. Quantum measuring processes of continuous observables. Journal of Mathematical Physics, 25(1):79-87, 1984. DOI: $10.1063 / 1.526000$.

[6] P. Liuzzo Scorpo, A. Cuccoli, and P. Verrucchi. Parametric description of the quantum measurement process. EPL (Europhysics Letters), 111(4):40008, 2015. DOI: 10.1209/0295-5075/111/40008.

[7] T. Heinosaari and M. Ziman. The Mathematical Language of Quantum Theory: From Uncertainty to Entanglement. Cambridge University Press, 2012. DOI: 10.1017/CBO9781139031103.

[8] Laurence G. Yaffe. Large $n$ limits as classical mechanics. Rev. Mod. Phys., 54:407-435, 1982. DOI: 10.1103/RevModPhys.54.407.

[9] D. Braun, F. Haake, and W.T. Strunz. Universality of decoherence. Phys. Rev. Lett., 86:2913-2917, 2001. DOI: 10.1103/PhysRevLett.86.2913.

[10] G. Chiribella and G.M. D'Ariano. Quantum information becomes classical when distributed to many users. Phys. Rev. Lett., 97:250503, 2006. DOI: 10.1103/PhysRevLett.97.250503.

[11] F. Galve, R. Zambrini, and S. Maniscalco. Non-markovianity hinders quantum darwinism. Scientific Reports, 6:19607, 2016. DOI: 10.1038/srep19607.

[12] G.L. Giorgi, F. Galve, and R. Zambrini. Quantum darwinism and non-markovian dissipative dynamics from quantum phases of the spin- $1 / 2 \quad x x$ model. Phys. Rev. A, 92:022105, 2015. DOI: 10.1103/PhysRevA.92.022105.

[13] L. Rigovacca, A. Farace, A. De Pasquale, and V. Giovannetti. Gaussian discriminating strength. Phys. Rev. A, 92:042331, 2015. DOI: 10.1103/PhysRevA.92.042331.

[14] P.A. Knott, T. Tufarelli, M. Piani, and G. Adesso. Generic emergence of objectivity of observables in infinite dimensions. Phys. Rev. Lett., 121:160401, 2018. DOI: 10.1103/PhysRevLett.121.160401.

[15] J. K. Korbicz, E. A. Aguilar, P. Ćwikliński, and P. Horodecki. Generic appearance of objective results in quantum mea- 
surements. Phys. Rev. A, 96:032124, 2017. DOI: 10.1103/PhysRevA.96.032124.

[16] G. Pleasance and B.M. Garraway. Application of quantum darwinism to a structured environment. Phys. Rev. A, 96:062105, 2017. DOI: 10.1103/PhysRevA.96.062105.

[17] P. Busch, P.J. Lahti, and P Mittelstaedt. The quantum theory of measurement. Springer-Verlag, Berlin, 1996. DOI: 10.1007/978-3-540-37205-9.

[18] D. Calvani, A. Cuccoli, N. I. Gidopoulos, and P. Verrucchi. Parametric representation of open quantum systems and crossover from quantum to classical environment. Proceedings of the National Academy of Sciences, 110(17):6748-6753, 2013. DOI: 10.1073/pnas.1217776110.

[19] D. Calvani. The Parametric Representation of an Open Quantum System. PhD thesis, Università degli Studi di Firenze, 2012.

[20] D. Calvani, A. Cuccoli, N. I. Gidopoulos, and P. Verrucchi. Dynamics of open quantum systems using parametric representation with coherent states. Open Systems \& Information Dynamics, 20(3):1340002, 2013. DOI: $10.1142 / \mathrm{S} 1230161213400027$.

[21] P. Liuzzo Scorpo, A. Cuccoli, and P. Verrucchi. Getting information via a quantum measurement: The role of decoherence. Int. J. Theor. Phys., 54(12):4356-4366, 2015. ISSN 1572-9575. DOI: $10.1007 / \mathrm{s} 10773-015-2548$ 8.

[22] C. Foti, A. Cuccoli, and P. Verrucchi. Quantum dynamics of a macroscopic magnet operating as an environment of a mechanical oscillator. Phys. Rev. A, 94:062127, 2016. DOI: 10.1103/PhysRevA.94.062127.

[23] M.A.C. Rossi, C. Foti, A. Cuccoli, J. Tra- pani, P. Verrucchi, and M.G.A. Paris. Effective description of the short-time dynamics in open quantum systems. Phys. Rev. A, 96:032116, 2017. DOI: 10.1103/PhysRevA.96.032116.

[24] W.M. Zhang, D.H. Feng, and R. Gilmore. Coherent states: Theory and some applications. Rev. Mod. Phys., 62:867-927, 1990. DOI: 10.1103/RevModPhys.62.867.

[25] A.M. Perelomov. Coherent states for arbitrary Lie group. Communications in Mathematical Physics, 26(3):222-236, 1972. ISSN 0010-3616. DOI: 10.1007/BF01645091.

[26] E. H. Lieb. The classical limit of quantum spin systems. Communications in Mathematical Physics, 31(4):327-340, 1973. DOI: 10.1007/BF01646493.

[27] S. Gnutzmann and M. Kus. Coherent states and the classical limit on irreducible $\mathrm{su}(3)$ representations. Journal of Physics A: Mathematical and General, 31(49):9871, 1998. DOI: $10.1088 / 0305-4470 / 31 / 49 / 011$.

[28] L. Querini. How quantum dynamics shape macroscopic evidences. Master thesis, University of Florence, 2016.

[29] C. Foti. On the macroscopic limit of quantum systems. $\mathrm{PhD}$ thesis, Università degli Studi di Firenze, 2019.

[30] M. Schlosshauer. Decoherence and the Quantum-To-Classical Transition. The Frontiers Collection. Springer, 2007. DOI: 10.1007/978-3-540-35775-9.

[31] F. A. Berezin. Models of gross-neveu type are quantization of a classical mechanics with nonlinear phase space. Comm. Math. Phys., 63(2):131-153, 1978. DOI: 10.1007/BF01220849. 


\section{A From Ozawa's model to the measure-and-prepare map}

Given a projective measurement with measurement operators $\{|\pi\rangle\langle\pi|\}$ acting on $\mathcal{H}_{\Gamma}$, its dynamical description according to the Ozawa's model is defined by the propagator $\exp \left\{-i t \hat{H}^{\mathrm{M}}\right\}$, with

$$
\hat{H}^{\mathrm{M}}=g \hat{O}_{\Gamma} \otimes \hat{O}_{\Xi}
$$

where $\hat{O}_{\Gamma}=\sum_{\pi} \omega_{\pi}|\pi\rangle\langle\pi|$ is the measured observable, while $\hat{O}_{\Xi}$ is the operator on $\mathcal{H}_{\Xi}$ conjugate to the pointer observable [30]. The resulting, measure-like, dynamics is such that decoherence of $\rho_{\Gamma}(t)$ w.r.t. the basis $\{|\pi\rangle\}$ implies $\rho_{\Xi}(t)=\sum_{\pi}\left|a_{\pi}\right|^{2}\left|\Xi_{t}^{\pi}\right\rangle\left\langle\Xi_{t}^{\pi}\right|$ with $\left\langle\Xi_{t}^{\pi} \mid \Xi_{t}^{\pi^{\prime}}\right\rangle=\delta_{\pi \pi^{\prime}}$ and $a_{\pi}$ such that $|\Gamma(0)\rangle=\sum_{\pi} a_{\pi}|\pi\rangle$, and viceversa. Here $t$ indicates any time prior the output production when decoherence has already occurred. This dynamics defines a CPTP map $\mathcal{E}^{\mathrm{M}}$ via

$$
|\Gamma\rangle\left\langle\Gamma\left|=\sum_{\pi \pi^{\prime}} a_{\pi} a_{\pi^{\prime}}^{*}\right| \pi\right\rangle\left\langle\left.\pi^{\prime}\left|\underset{\mathcal{E}^{\mathrm{M}}}{\longrightarrow} \rho_{\Xi}=\sum_{\pi}\right| a_{\pi}\right|^{2} \mid \Xi^{\pi}\right\rangle\left\langle\Xi^{\pi}\right|
$$

referred to as measure-and-prepare $(\mathrm{m} \& \mathrm{p})$ map in the literature. Notice that what characterizes $\mathcal{E}^{\mathrm{M}}$ as a m\&p map is not the diagonal form of the output state $\rho_{\Xi}$, but rather the fact that its eigenvalues are constant and exclusively depend on the input state $|\Gamma\rangle\langle\Gamma|$.

\section{B Large- $N$ as classical limit}

In order to define the classical limit of a quantum theory $\mathcal{Q}$ it is first necessary to identify a parameter $k$, usually dubbed "quanticity parameter", such that $\mathcal{Q}$ transforms into a classical theory $\mathcal{C}$ as $k$ vanishes. By "transform" it is meant that a formal relation is set between Hilbert and phase spaces, Lie and Poisson brackets, Hamiltonian operators and functions. Consequently, the large- $N$ limit of $\mathcal{Q}$ implies a classical behaviour of the macroscopic system it describes IF $N \rightarrow \infty$ implies $k \rightarrow 0$. On the other hand, in order for this being the case it proves sufficient that GCS $\{|\omega\rangle\}$ for $\mathcal{Q}$ exist and feature some specific properties $[31,8]$. Amongst these, particularly relevant to this work is that

$$
\lim _{k \rightarrow 0} k\left[\ln \left|\left\langle\omega^{\prime} \mid \omega\right\rangle\right|\right] \leq 0
$$

where the equality holds iff $\omega=\omega^{\prime}$, and the property implies the limit exists. From the above property it follows 4

$$
\lim _{k \rightarrow 0} \frac{1}{k}\left|\left\langle\omega \mid \omega^{\prime}\right\rangle\right|^{2}=\delta\left(\omega-\omega^{\prime}\right),
$$

which is a most relevant properties of GCS, namely that they become orthogonal in the classical limit.

It is worth mentioning that if $\mathcal{Q}$ features a global symmetry (also dubbed "supersymmetry" in the literature), GCS can be explicitly constructed and shown to feature the properties ensuring that the large- $N$ limit is indeed a classical one [8]. However, whether the existence of one such symmetry be a necessary condition for a system to behave classically in the large- $N$ limit is not proven, although all of the known physical theories, be they vector-, matrix-, or gauge-theories, confirm the statement (see Sec.VII of Ref. [8] for a thorough discussion about this point). Incidentally, we believe the above supersymmetry be essential in defining what a macroscopic observer should actually be in order for Quantum Darwinism to occur, in a way similar to that discussed in Ref. [10] in the specific case of a quantum theory for $N$ distinguishable particles with permutation global symmetry.

\section{Overlap between GCS and elements of an orthonormal basis in the large- $N$ limit}

One of the output of the GCS construction, and key-ingredient for their use, is the invariant measure $d \mu(\hat{\omega})$ entering the identity resolution Eq.(12). It is demonstrated [8] that in order for such resolution

${ }^{4}$ We use the Dirac- $\delta$ representation $\delta(x-y)=\lim _{\epsilon \rightarrow 0}(1 / \epsilon) \exp \left\{(x-y)^{2} / \epsilon\right\}$. 
to keep holding for whatever value of the quanticity parameter $k$ it must be $d \mu(\omega)=c_{k} d m(\omega)$, with $c_{k}$ a constant on $\mathcal{M}$ that depends on the normalization of the group-measure $d \mu(\hat{\omega})$ and should be computed on a case-by-case basis. However, normalization of GCS is guaranteed by construction, and hence, via Eq.(12),

$$
\langle\omega \mid \omega\rangle=\int_{\mathcal{M}} c_{k} d m\left(\omega^{\prime}\right)\left|\left\langle\omega \mid \omega^{\prime}\right\rangle\right|^{2}=1, \forall|\omega\rangle ;
$$

Furthermore, from Eq.(27) it follows $\left|\left\langle\omega \mid \omega^{\prime}\right\rangle\right|^{2} \rightarrow k \delta\left(\omega-\omega^{\prime}\right)$ as $k$ vanishes, and hence

$$
\lim _{k \rightarrow 0} c_{k} k \int_{\mathcal{M}} d m\left(\omega^{\prime}\right) \delta\left(\omega-\omega^{\prime}\right)=1,
$$

which implies $c_{k}=\frac{1}{k}$, as readily verified in those cases where an explicit form of GCS is available. The fact that $c_{k}$ is independent of $\omega$ and goes like $\frac{1}{k}$ for vanishing $k$, enforces

$$
\lim _{k \rightarrow 0} \int_{\mathcal{M}} \frac{1}{k} d m(\omega)\left\langle\xi^{\prime} \mid \omega\right\rangle\left\langle\omega \mid \xi^{\prime \prime}\right\rangle=\delta_{\xi^{\prime} \xi^{\prime \prime}}
$$

to hold for whatever pair $\left(\left|\xi^{\prime}\right\rangle,\left|\xi^{\prime \prime}\right\rangle\right)$ of orthonormal states: as neither $d m(\omega)$ nor $\mathcal{M}$ depend on $k$, this is only possible if the two overlaps entering the integral are never simultaneously finite on $\mathcal{M}$ or, more precisely, on a set of finite measure. In other terms, Eq.(30) implies Eq.(20), and viceversa (which is trivial). 